\title{
Risk factors for catheter related bloodstream infections in patients on home parenteral nutrition: An audit from a tertiary center in 2017
}

Konstantinos C. Fragkos, Krista Murray, Sarah Obbard, Thomas Shepherd, John Barragry, Akudo Nwaogu, Jennifer Rogers, Shola Ajibodu, Niamh Keane, Margaret MacRae, Pinal Patel, Shameer Mehta, Simona Di Caro, Farooq Rahman

Intestinal Failure Service, University College London Hospitals NHS Foundation Trust, London, United Kingdom

\begin{abstract}
Rationale: Incidence of catheter related bloodstream infections (CRBSIs) is often a surrogate marker of the overall quality of care in home parenteral nutrition (HPN) patients. Our aim was to compare CRBSI rate between a clinical definition and CDC/ESPEN definition and identify risk factors.
\end{abstract}

Methods: A single-centre intestinal failure database was used. The study period was between 1 January 2017 and 31 December 2017. A CRBSI was defined clinically as generalized systemic inflammatory symptoms with a positive blood culture (central and/or peripheral) with exclusion of other detectable foci. Statistical analyses included percentages, means and univariate Poisson regression for risk factor identification.

Results: There were 143 active HPN patients in 2017 (females 69.2\%, 53.8 \pm 16.6 years). Short bowel syndrome was present in $49.6 \%$ patients followed by mechanical obstruction in $23.1 \%$. Tunnelled lines were present in $46.2 \%$ and peripherally inserted central catheter lines in 53.8\%. Single and double lumen lines were present in $18.4 \%$ and $80.9 \%$, respectively. 38 CRBSIs were observed in 35231 catheter days. The catheter was left in situ in $21 \%$ and replaced or removed in $79 \%$. The overall CRBSI incidence rate (episodes/1000 catheter days) was 0.23 (95\% Cl 0.11-0.45) (CDC/ESPEN) vs 1.08 (95\% Cl 0.78-1.48) (UCLH modified CRBSI) (difference 0.85, p<0.0001). 8 out of 38 CRSBI (21\%) satisfied CDC/ESPEN criteria. 33 Infections (87\%) were due to a single microorganism and 5 (13\%) due to more than one microorganisms. $84 \%$ were due to gram positive bacteria, $34 \%$ were due to gram negative bacteria and $8 \%$ due to candida. Higher incidence of CRBSIs (UCLH defintion) was non-significantly noted in patients with intestinal fistula (4.51), tunnelled lines (1.36), having PN (1.23), independent nursing (1.83) and taurolidine (1.73).

Conclusion: A significant variation is noted in CRBSI incidence according to definition. This affects significance in risk factors. This should be taken into account when comparing rates between centres and formulating national surveillance plans of intestinal failure services.

Disclosure of Interest: None declared. 